\title{
Fact Sheet: Mayaro Virus ${ }^{1}$
}

\author{
Lawrence Reeves, Lindsay P. Campbell ${ }^{2}$
}

\section{Introduction}

Mayaro virus (MAYV) is an arthropod-borne virus (arbovirus) that can be transmitted from mosquitoes to humans. The geographic distribution of Mayaro virus is currently expanding north from South and Central America into the Caribbean Islands, which means that under the right conditions, this virus could one day become important to Florida. This publication is intended to provide information about MAYV to researchers and stakeholders in mosquito control and public health professions and to the general public.

\section{What is Mayaro Virus?}

MAYV is a poorly known zoonotic arbovirus (a virus of non-human animals, spread by arthropod vectors, that occasionally is transferred to humans) in the genus Alphavirus that is transmitted to animals and humans through the bite of a mosquito. Mayaro virus was discovered in 1954 on the islands of Trinidad and Tobago (Causey and Maroja 1957) and is the causative agent of Mayaro virus disease (MAYVD). MAYV occurs mainly in regions of Central and South America and is largely associated with forested areas. More recently, the virus was reported from Haiti on the Caribbean island of Hispaniola (Blohm et al. 2019). MAYV is considered an emerging infectious disease because of its recent expansion into new geographic regions. Although local transmission of MAYV has not occurred in the United States, mosquito species known to be capable of transmitting the virus are present in the southeastern United States
(Reeves et al. 2020), suggesting the potential for MAYVD to occur in this region.

Since the initial discovery of MAYV in Trinidad and Tobago, MAYVD has been diagnosed in multiple countries in Central and South America, and the virus has been isolated from animals or mosquitoes in additional countries where human disease has not yet been reported (Figure 1, Caicedo et al. 2020).

\section{Transmission Cycle}

The transmission cycle of MAYV is poorly characterized. MAYV is thought to be maintained in the natural environment through a transmission cycle that involves forestdwelling mosquitoes and vertebrate animals, likely mammals and birds (Mackay and Arden 2016). Occasionally, MAYV "spills over" to humans. Such spill-over events are most often associated with human activities within forested areas or near forest edges (Mota et al. 2015). However, MAYV has the potential to establish an urban transmission cycle by which mosquitoes that are competent at transmitting the virus feed on infected animals or humans and then transmit the virus to other humans (Mackay and Arden 2016).

Like other arboviruses, MAYV is transmitted between vertebrate host animals by the bite of an infected female mosquito. Mosquito species vary in their vector competency (ability to become infected with and transmit a particular virus). Female mosquitoes that are competent

1. This document is ENY-2074, one of a series of the Entomology and Nematology Department, UF/IFAS Extension. Original publication date December 2021. Visit the EDIS website at https://edis.ifas.ufl.edu for the currently supported version of this publication.

2. Lawrence Reeves, assistant professor; and Lindsay P. Campbell, assistant professor, Entomology and Nematology Department; UF/IFAS Florida Medical Entomology Laboratory.

The Institute of Food and Agricultural Sciences (IFAS) is an Equal Opportunity Institution authorized to provide research, educational information and other services

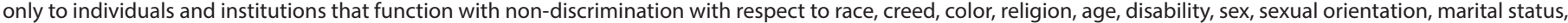

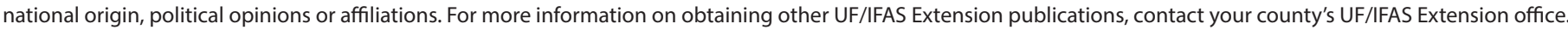
U.S. Department of Agriculture, UF/IFAS Extension Service, University of Florida, IFAS, Florida A \& M University Cooperative Extension Program, and Boards of County Commissioners Cooperating. Nick T. Place, dean for UF/IFAS Extension. 
vectors for MAYV become infected when they feed from the blood of a MAYV-infected vertebrate host. Within a competent mosquito, the infection spreads from the gut to the salivary glands. When this happens, the mosquito may then infect subsequent vertebrate hosts upon which it feeds. These vertebrate hosts may become infected with MAYV, amplify the infection, and infect subsequent competent mosquitoes that feed upon them, thereby contributing toward a cycle of transmission.

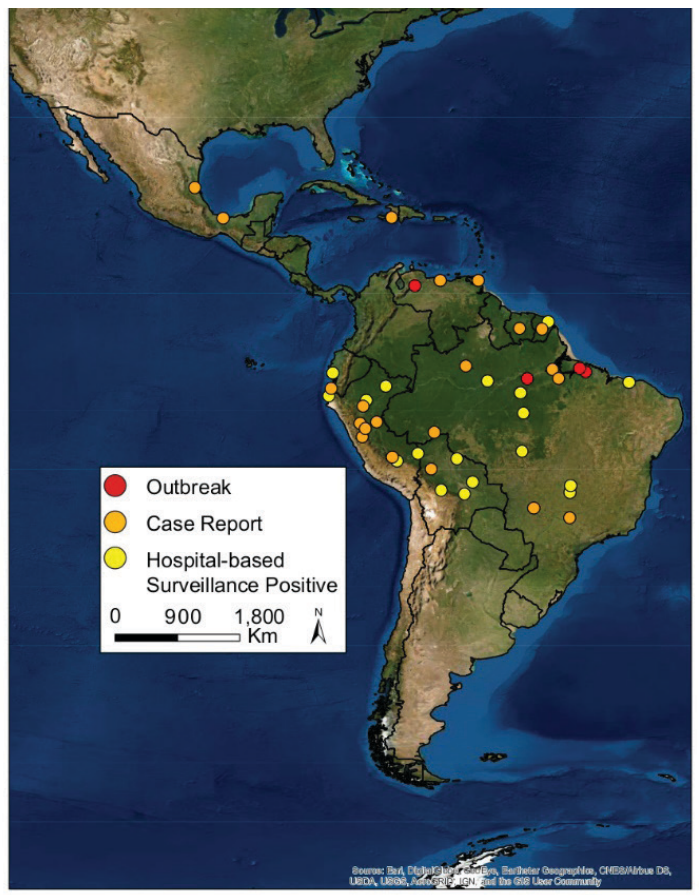

Figure 1. Map adapted from Caicedo et al. 2020 that compiled confirmed MAYVD outbreaks, case reports, and hospital-based surveillance from a systematic review of the scientific literature. Case reports were confirmed through one of three molecular tests: viral isolation, presence of Immunoglobulin M (IgM) antibodies that indicate a current or recent infection, or PCR. Hospital-based surveillance included testing of patients exhibiting a fever using a combination of PCR and testing for the presence of IgM antibodies. Image Basemap source: Esri, DigitalGlobe, GeoEye, Earthstar Geographics, CNES/Airbus DS, USDA, USGS, AeroGRID, and the GIS User Community.

Credits: Lindsay P. Campbell, UF/IFAS

The mosquito species most important to MAYV transmission are unknown. The mosquito Haemagogus janthinomys (Figure 2) was found to be infected with MAYV during several MAYV outbreaks in Brazil, and in these cases was suspected to be the primary vector responsible for spillover of the virus into humans (Hoch et al. 1981). Haemagogus janthinomys is a diurnal forest-dwelling mosquito that occurs across a wide distribution from Honduras south through the northern half of South America. Eggs are laid and larvae develop in water-holding treeholes. Adults are associated with the canopy, but host-seeking females will descend to ground level to feed, particularly in areas where the canopy has been disturbed by deforestation. Female Haemagogus janthinomys feed on a broad range of vertebrate hosts, especially birds and mammals, including humans, non-human primates, and rodents (Alencar et al. 2005). In addition to MAYV, this mosquito is an important vector for yellow fever virus, and Ilheus virus has been isolated from specimens collected in Panama.

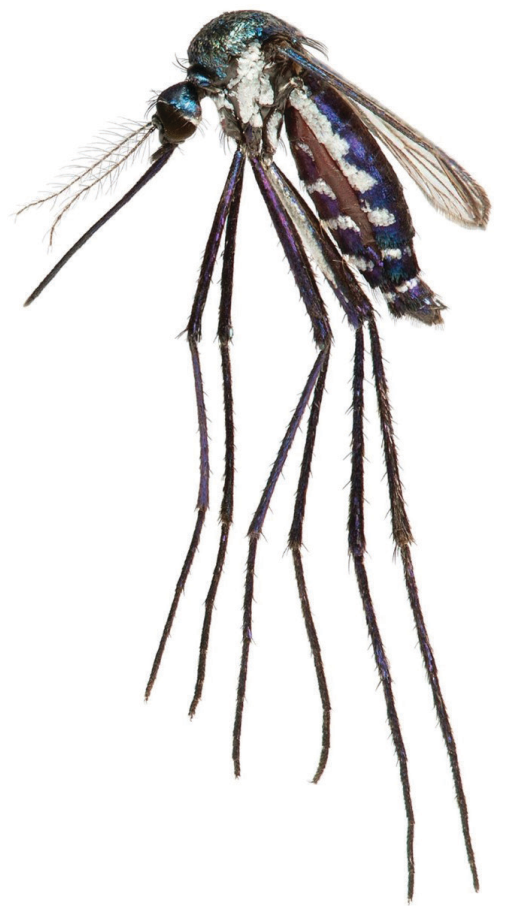

Figure 2. Adult female Haemagogus janthinomys, an expected important vector of Mayaro virus, collected on Ilha Grande, Rio de Janeiro, Brazil.

Credits: Lawrence Reeves, UF/IFAS

In laboratory experiments using mosquito species collected in Trinidad, the mosquito Aedes scapularis (Figure 3) was capable of transmitting MAYV to new vertebrate hosts (Aitken and Anderson 1959). Aedes scapularis has an exceptionally wide distribution that spans from the Rio Grande Valley of Texas, south to central Argentina (Arnell 1976). Females take blood from mammals, birds, and reptiles, and in some areas the species has adapted to human-dominated rural and urban landscapes where it readily enters buildings and feeds from humans indoors (Arnell 1976). Larval habitats include temporary ground waters and pools at the margins of streams and other water bodies, among others. Established populations of Aedes scapularis were recently detected in peninsular Florida, where the species is non-native. Aedes scapularis is now widespread on the southeastern coast of Florida in MiamiDade and Broward Counties (Reeves et al. 2020), and ecological niche modeling suggests that large areas of the Florida Peninsula, particularly along the Gulf and Atlantic Coastlines, are environmentally suitable for the species (Campbell et al. 2021). 


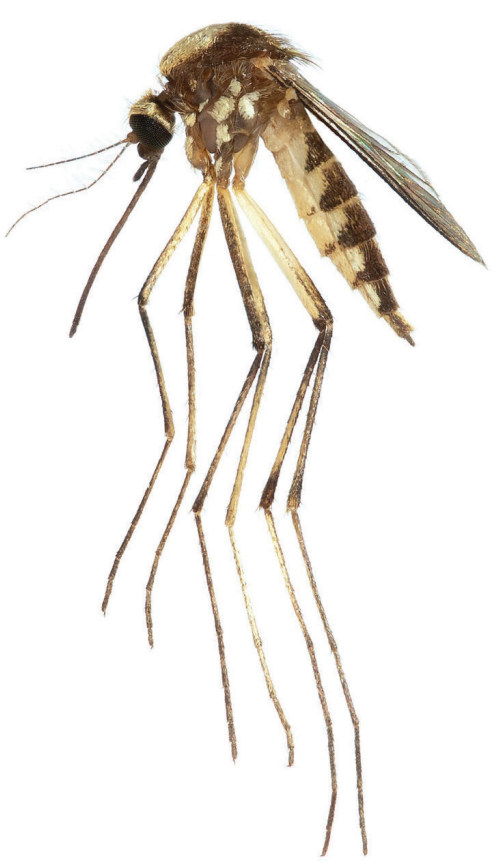

Figure 3. Adult female Aedes scapularis, a potential vector of Mayaro virus, collected in Miami-Dade County, Florida.

Credits: Lawrence Reeves, UF/IFAS

Haemagogus janthinomys is the only species currently implicated as a vector during MAYV outbreaks, though other species may be important as vectors during outbreaks or in maintaining the virus in nature.

Various other mosquito species have been found naturally infected with MAYV, including Mansonia venezuelensis, Culex vomerifer, and Psorophora ferox (Mackay and Arden 2016). The latter species occurs in woodland areas throughout the southeastern United States, as well as much of Central and South America (Darsie and Ward 2005). Aedes aegypti mosquitoes, also known as the yellow fever mosquito, have also been found naturally infected with MAYV in South America, and laboratory studies have shown that this mosquito is capable of transmitting the virus to host species but that transmission rates are low (Long et al. 2011; Serra et al. 2016; Wiggins et al. 2018). In the Americas, Ae. aegypti is a human-adapted mosquito species, feeding primarily on humans while maintaining close proximity to human environments, which poses some risk for MAYV transmission to humans, but it is expected that this species is not likely to sustain transmission of MAYV in the human population (Wiggins et al. 2018).

Vertebrate host animals vary in their ability to become infected with particular arboviruses, and, once infected, in their ability to infect new mosquito vectors. Some vertebrates are "dead end hosts." These hosts may become infected and experience disease, but the virus cannot replicate to a sufficient level within them to infect subsequent mosquito vectors that feed from them. Others are "reservoir hosts." These hosts are susceptible to infection, may or may not experience disease, and allow sufficiently high levels of virus replication to infect any subsequent competent mosquito vectors.

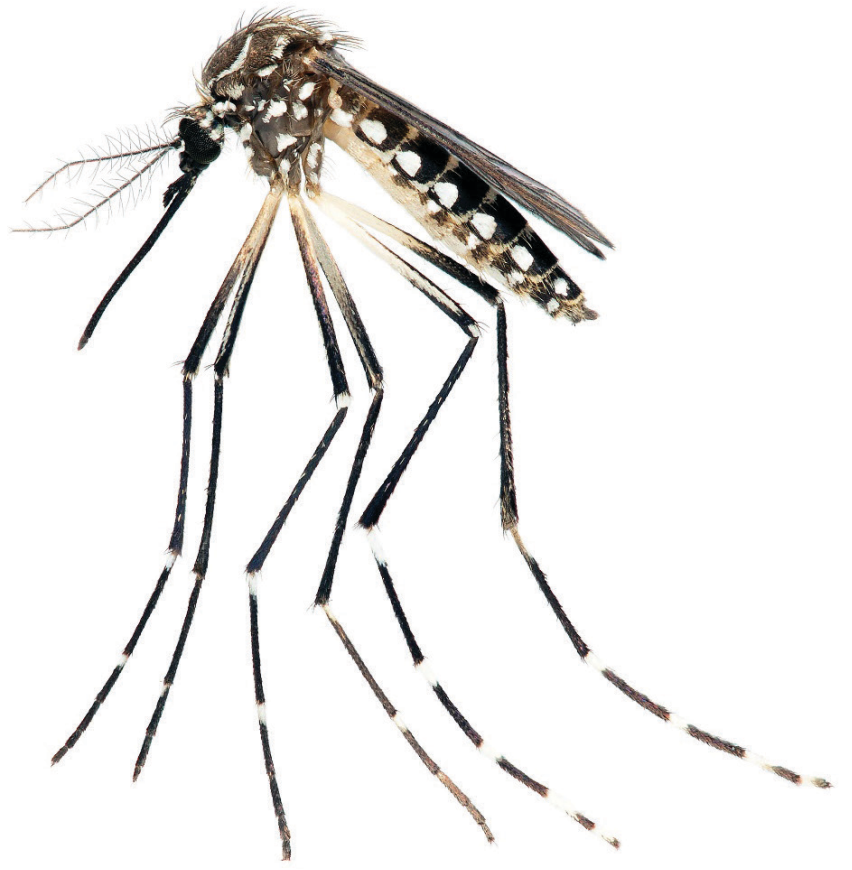

Figure 4. Aedes aegyptimosquito, a potential vector of Mayaro virus, collected in Indian River County, Florida.

Credits: Lawrence Reeves, UF/IFAS

The vertebrate host species that are the most important to the maintenance of MAYV transmission cycles as reservoir hosts have not yet been identified. A number of mammal and bird species have been found to be MAYV seropositive; they have antibodies indicating a previous MAYV infection. In Brazil, about $30 \%$ of silvery marmosets were seropositive for past infections of MAYV, and laboratory experiments confirmed their ability to serve as hosts (Hoch et al. 1981). In French Guiana and Panama, several species of howler monkeys, tamarinds, and other monkeys were seropositive for past infections of MAYV (Mackay and Arden 2016). MAYV and evidence of MAYV infections have also been detected in birds, including an orchard oriole in Louisiana and approximately $30 \%$ of tested ground doves during an outbreak in Brazil. Various other animals have been found to be seropositive for MAYV, including sloths, opossums, armadillos, coatimundis, agoutis, and horses (Mackay and Arden 2016).

\section{Symptoms}

Symptoms of MAYVD can be difficult to distinguish from other arboviral disease symptoms and may include fever, joint pain, rash, headache, chills, and eye pain and/or light sensitivity (Mota et al. 2015). As in chikungunya disease, 
long-term joint pain lasting up to approximately one year is possible (Acosta-Ampudia et al. 2018). Although relatively rare, MAYVD can result in heart problems, neurological complications, and death.

\section{Diagnosis}

A person infected with MAYV may exhibit a fever, joint pain, or other symptoms that prompts a doctor to consider whether an arbovirus may be responsible for illness. If the person is residing in or has traveled recently to a region where MAYV occurs, the doctor may test for the virus, along with additional arboviruses known to circulate in the region. A set of one or more serological or molecular tests can be used to confirm a MAYV infection (Mota et al. 2015). These methods include virus isolation, reverse transcriptase PCR, (a test based on the presence or absence of MAYV genetic material), or a serological test that identifies the presence of MAYV IgM antibodies that can indicate the presence of or recent infection with the virus (Acosta-Ampudia et al. 2018).

\section{Treatment}

Supportive care is provided to patients with MAYVD, including medication for joint pain and fever reduction. Drugs developed to speed recovery of symptoms in chikungunya patients have been shown to be effective for some symptoms of MAYVD (Acosta-Ampudia et al. 2018).

\section{Challenges}

MAYV symptoms in humans are most often mild, but more severe symptoms similar to other arboviral illnesses including dengue, chikungunya, and Zika, can occur. These similarities can lead to clinical misdiagnoses (Mota et al. 2015). Humans can become infected with more than one arbovirus at the same time, which can also lead to misdiagnoses or underreporting (Rodriguez-Morales et al. 2016). MAYV is closely related to other alphaviruses, for example chikungunya virus, and cross-reactivity in molecular diagnostic tests can occur, resulting in a false positive test (Acosta-Ampudia et al. 2018).

\section{Prevention}

Currently, a vaccine is not available for MAYV, but scientists are in the process of developing and testing vaccines (Acosta-Ampudia et al. 2018). The best protection against acquiring MAYV or any mosquito-borne disease is to limit exposure to mosquitoes and protect against their bites: apply mosquito repellent when working or recreating outdoors, wear long sleeved shirts and pants to protect exposed skin from mosquito bites, and eliminate standing water around your home or property. Individuals living in or visiting countries where MAYV is known to circulate should be particularly vigilant and take precautions to limit mosquito exposure when near forests or forest edges, where risk of MAYV infection is particularly high.

\section{References}

Acosta-Ampudia, Y., D. M. Monsalve, Y. Rodríguez, Y. Pacheco, J. M. Anaya, and C. Ramírez-Santana. 2018. “Mayaro: An Emerging Viral Threat?” Emerging Microbes \& Infections 7:1-11. https://doi.org/10.1038/ s41426-018-0163-5

Aitken, T., and C. Anderson. "Virus Transmission Studies with Trinidadian Mosquitoes: II. Further Observations." 1959. American Journal of Tropical Medicine and Hygiene 8:41-45. https://doi.org/10.4269/ajtmh.1959.8.41

Alencar, J., E. S. Lorosa, N. Degallier, N. M. Serra-Freire, J. B. Pacheco, and A. E. Guimaraes. 2005. "Feeding Patterns of Haemagogus janthinomys (Diptera: Culicidae) in Different Regions of Brazil." Journal of Medical Entomology 42:981-985. https://doi.org/10.1093/jmedent/42.6.981

Arnell, J. H. 1976. "Mosquito Studies XXXIII. A Revision of the Scapularis Group of Aedes (Ochlerotatus)." Contributions of the American Entomological Institute 13:1-144.

Blohm, G., M. A. Elbadry, C. Mavian, C. Stephenson, J. Loeb, S. White, T. Telisma, et al. 2019. "Mayaro as a Caribbean Traveler: Evidence for Multiple Introductions and Transmission of the Virus into Haiti." International Journal of Infectious Diseases 87:151-153. https://doi.org/10.1016/j. ijid.2019.07.031

Caicedo, E. Y., K. Charniga, A. Rueda, I. Dorigatti, Y. Mendez, A. Hamlet, J. P. Caarrera, and Z. M. Cucunubá. "The Epidemiology of Mayaro Virus in the Americas: A Systematic Review and Key Parameter Estimates for Outbreak Modelling." medRxiv preprint doi: https://doi. org/10.1101/2020.12.10.20247296

Campbell, L. P., N. D. Burkett-Cadena, E. Miqueli, I. Unlu, K. Sloyer, J. Medina, C. Vasquez, W. Petrie, and L. E. Reeves. 2021. "Potential Distribution of Aedes (Ochlerotatus) scapularis (Diptera: Culicidae): A Vector Mosquito New to the Florida Peninsula." Insects 12:213. https://doi.org/10.3390/ insects 12030213 
Causey, O. R., and O. M. Maroja. 1957. "Mayaro Virus: A New Human Disease Agent." American Journal of Tropical Medicine and Hygiene 6:1017-1023. https://doi. org/10.4269/ajtmh.1957.6.1017

Darsie, R. F., and R. A. Ward. 2005. Identification and Geographical Distribution of the Mosquitoes of North America, North of Mexico. University Press of Florida, Gainesville, FL.

Hoch, A. L., N. E. Peterson, J. W. LeDuc, and F. P. Pinheiro. 1981. "An Outbreak of Mayaro Virus Disease in Belterra, Brazil. III. Entomological and Ecological Studies." American Journal of Tropical Medicine and Hygiene 30:689-698. https://doi.org/10.4269/ajtmh.1981.30.689

Long, K., S. Ziegler, S. Thangamani, N. Hausser, T. Kochel, S. Higgs, and R. B. Tesh.2011. "Experimental Transmission of Mayaro Virus by Aedes aegypti." The American Journal of Tropical Medicine and Hygiene. 85:750-7. https://doi. org/10.4269/ajtmh.2011.11-0359

Mackay, I. M., and K. E. Arden. 2016. "Mayaro Virus: A Forest Virus Primed for a Trip to the City?" Microbes and Infection 18:724-734. https://doi.org/10.1016/j. micinf.2016.10.007

Mota, M. T. O., M. R. Ribeiro, D. Vedovello, and M. L. Nogueira. 2015. "Mayaro Virus: A Neglected Virus of the Americas." Future Virology 10:1109-1122. https://doi. org/10.2217/fvl.15.76

Reeves, L. E., J. Medina, E. Miqueli, K. E. Sloyer, W. Petrie, C. Vasquez, and N. D. Burkett-Cadena. 2020. "Establishment of Aedes (Ochlerotatus) scapularis (Diptera: Culicidae) in Mainland Florida, with Notes on the Ochlerotatus Group in the United States." Journal of Medical Entomology, tjaa250. https://doi.org/10.1093/jme/tjaa250

Rodriguez-Morales, A. J., W. E. Villamil-Gómez, and C. Franco-Paredes. 2016. "The Arboviral Burden of Disease Caused by Co-Circulation and Co-Infection of Dengue, Chikungunya and Zika in the Americas." Travel Medicine and Infectious Disease 14:177-179. https://doi.org/10.1016/j. tmaid.2016.05.004

Serra, O., B. Cardoso, A. Ribeiro, F. dos Santos, and R. Slhessarenko. 2016. "Mayaro Virus and Dengue Virus 1 and 4 Natural Infection in Culicids from Cuiabá, State of Mato Grosso, Brazil." Mem Inst Oswaldo Cruz. 111 (1):20-9. https://doi.org/10.1590/0074-02760150270
Wiggins, K., B. Eastmond, and B. A. Alto. 2018. "Transmission Potential of Mayaro Virus in Florida Aedes aegypti and Aedes albopictus Mosquitoes." Medical and Veterinary Entomology 32:436-442. https://doi.org/10.1111/mve.12322 